\title{
Low Count Detection for EELS Spectrum by Reducing CCD Read-out Noise
}

\author{
Mitsutaka Haruta $^{1}$, Yoshifumi Fujiyoshi ${ }^{1}$, Takashi Nemoto ${ }^{1}$, Akimitsu Ishizuka ${ }^{2}$, Kazuo Ishizuka ${ }^{2}$ and \\ Hiroki Kurata ${ }^{1}$ \\ ${ }^{1}$ Kyoto University, Uji, Kyoto, Japan, ${ }^{2}$ HREM Research Inc., Higashimatsuyama, Saitama, Japan
}

STEM-EELS is useful for examining local chemistry, electronic structure with high spatial resolution. In addition, recent developments in monochromated electron sources allow high-energy resolution. Thus, in principle, the atomic resolution electronic state analysis with high energy resolution is possible. However, it is not easy to obtain high signal-to-noise ( $\mathrm{SN}$ ) spectra with high spatial resolution without sample damage due to the limitations of practical experimental conditions. Therefore, multiple spectrum imaging (SI) measurements with low dose rate is one way of solving this problem [1,2]. However, in the case of extremely low count signal, the summation of spectra cannot improve SN ratio while it is using common acquisition procedure.

In the previous research, Bosman et al., reported a method to shift the spectrum during SI measurement to suppress the gain variation [3]. In addition, high-quality dark reference (HQD) subtraction was proposed by Hou [4], who obtained HQD by averaging 128 spectra. The GMS (Gatan Microscopy Suite) software [5] has the option of subtracting HQD from the experimental spectrum. However, a detailed discussion of the dark reference in EELS based on statistics has not yet been published. As it turns out, it appeared that the main component of noise in SI experiment was read-out noise, and the sampling number required to estimate the population mean of dark reference was statistically insufficient in the previous method. In the present contribution, we report a systematic statistical study on the reduction of CCD noise for EELS using our original method for the high-quality dark reference combined with a gain-averaging method. [6]

STEM-EELS experiments were carried out on a JEM-ARM200F (200 kV) using a GIF Quantum ERS (Gatan, Inc.). The Ti $L_{2,3}$-edge spectra were obtained for $\mathrm{SrTiO}_{3}$. The vertical hardware binning was 130. Moreover, in order to reduce the gain variation of the CCD, each spectrum of the dual EELS mode was shifted slightly during the SI measurement by using a prism shift, where both spectra of the dual EELS were shifted at the same time.

Figure 1 (a) shows a single frame Ti L2,3-edge obtained with a very low dose. In a single spectrum, it is impossible to recognize the Ti $L_{2,3}$-edge signal. On the other hand, Fig. 1(b) shows the spectra averaged over 12,000-frames after subtraction of our original ultra-high-quality dark reference (UHQD) which was estimated using 20,000 frames. In addition, the result of gain averaging after subtraction of UHQD shows a further improvement of the noise standard deviation (Fig. 1 (c)). The average peak intensity of the first peak of the $\mathrm{Ti}$ L3-edge after subtracting the power-law background was only 1.2 counts, which corresponds to 0.15 electrons per dwell time using the estimated conversion factor of our CCD (about 8 counts/electron for 200-keV electron). It is thereby demonstrated that a high-SN spectrum can be obtained even for a single-count core-loss signal. The present method would be useful for measuring low signal spectrum such as monochromated spectra and for radiation sensitive materials. [7]

\section{Acknowledgements}


We would like to thank Dr. Colin Trevor from Gatan R\&D for his valuable comments. This work was supported by JSPS KAKENHI Grant Numbers $17 \mathrm{H} 02739$ and $19 \mathrm{H} 02597$ and partly supported by the JSPS Core-to-Core Program (A) Advanced Research Networks
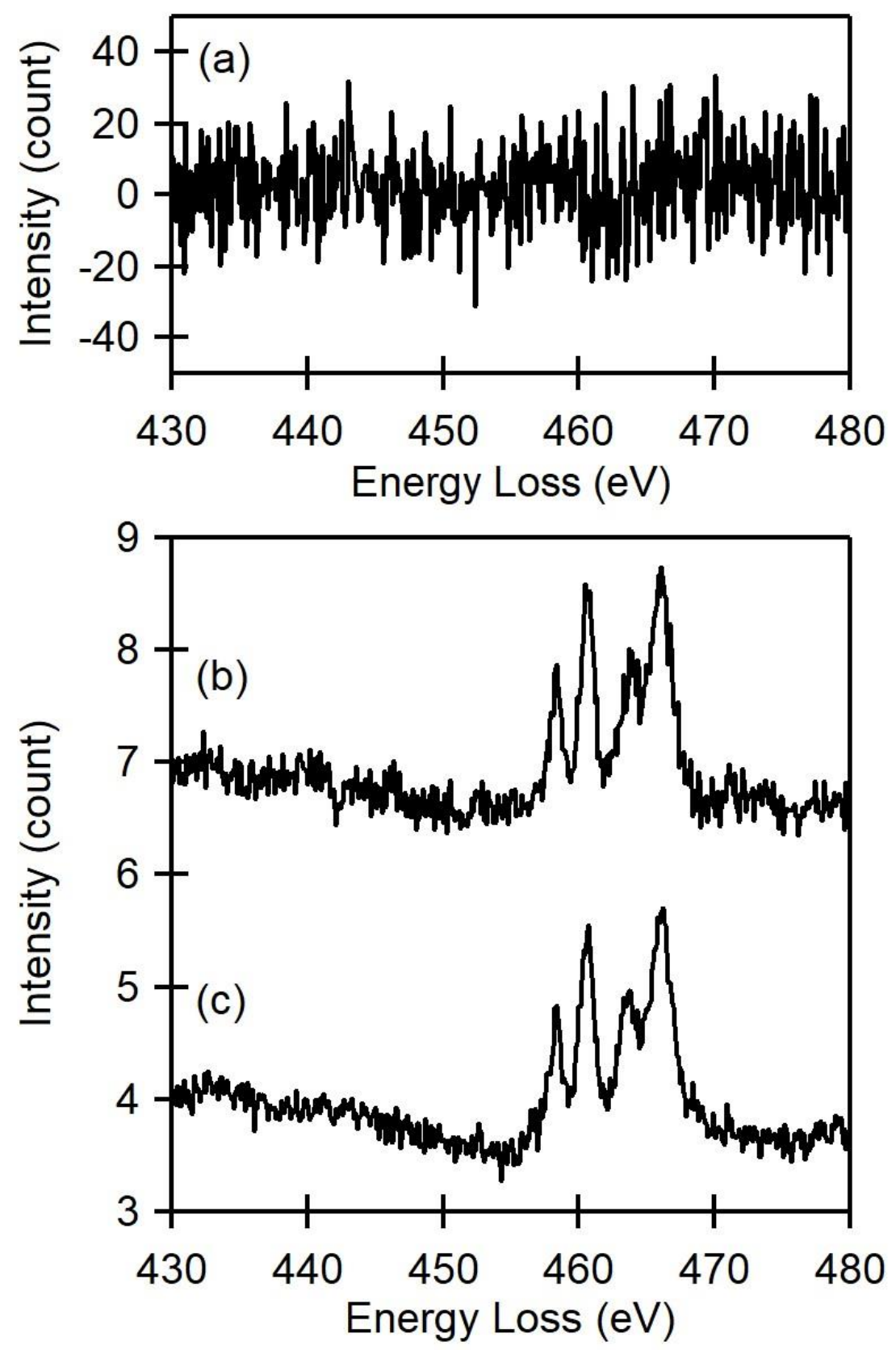

Figure 1. (a) A single-frame Ti L2,3-edge spectrum of SrTiO3 obtained with a very weak signal. (b) Spectra averaged over 12,000 frames without background subtraction after subtraction ultra-high-quality dark reference. The spectrum is vertically shifted by 3 counts. (c) The result of gain averaging after subtraction of ultra-high-quality dark reference. The spectral intensities (b) and (c) were normalized as a single spectrum.

References 
[1] L. Jones et al., Advanced Structural and Chemical Imaging 1, 8 (2015).

[2] L. Jones et al., Microscopy 67, i98-i113 (2018).

[3] M. Bosman et al., Ultramicroscopy 106, 1024-1032 (2006).

[4] V. D. Hou, Microsc. Microanal. 15, (S2), 226-227 (2009).

[5] Manual of Gatan Microscopy Suite (GMS), Gatan Inc..

[6] M. Haruta et al., Ultramicroscopy 207, 112827 (2019).

[7] M. Haruta et al., Phys. Rev. B 97, 205139 (2018). 